\title{
Enhancing Drought Tolerance of Wheat (Triticum aestivgum L.) Through Foliar Application of Proline and L-Triptophan
}

\author{
Asad Shabir ${ }^{b}$, Muhammad Saqib ${ }^{b}$, Maqshoof Ahmad ${ }^{c}$, Muhammad Latif ${ }^{b}$, \\ Syed Asad Hussain Bukharia*, Muhammad Qadir Ahmad ${ }^{\mathrm{d}}$, \\ Muhammad Dawood ${ }^{\mathrm{e}}$ and Muhammad Rashid ${ }^{\mathrm{f}}$ \\ ${ }^{a}$ Department of Agronomy, Bahauddin Zakariya University, Multan-60800, Pakistan \\ ${ }^{b}$ Department of Agronomy, University College of Agriculture and Environmental Sciences, \\ The Islamia University of Bahawalpur-63100, Pakistan \\ ${ }^{c}$ Department of Soil Sciences, University College of Agriculture and Environmental Sciences, \\ The Islamia University of Bahawalpur-63100, Pakistan \\ dDepartment of Plant Breeding and Genetics, Bahauddin Zakariya University, Multan-60800, Pakistan \\ ${ }^{\mathrm{e}}$ Department of Environmental Sciences, Bahauddin Zakariya University, Multan-60800, Pakistan \\ fDepartment of Agronomy, Lasbela University of Agriculture, Water and Marine Sciences, Uthal, Pakistan
}

(received May 15, 2017; revised December 3, 2018; accepted December 14, 2018)

\begin{abstract}
Limited water availability is one of the important abiotic factor affecting yield of wheat crop. Exogenous application of osmolytes is an important factor in reducing the stress due to water shortage. Keeping in view the role of proline and L-tryptophan (L-TRP) in stress alleviation, a study was carried out at the agricultural research area of the University College of Agriculture and Environmental Sciences, The Islamia University of Bahawalpur, Pakistan, during winter 2015-2016 to evaluate the impact of foliar applied proline and L-TRP on growth and photosynthetic efficiency of wheat grown under limited moisture supply. Drought stress was applied in three levels $\left(\mathrm{I}_{1}=\right.$ control, $\mathrm{I}_{2}=$ drought stress at tillering stage and $\mathrm{I}_{3}=$ drought stress at grain filling stage) while, foliar application of proline and L-TRP was done in six levels $\left[\mathrm{T}_{1}=\right.$ control; $\mathrm{T}_{2}=$ proline $(10 \mathrm{mM}) ; \mathrm{T}_{3}=$ proline $(20 \mathrm{mM}) ; \mathrm{T}_{4}=\mathrm{L}-\mathrm{TRP}\left(10^{-4} \mathrm{M}\right) ; \mathrm{T}_{5}=\mathrm{L}-\mathrm{TRP}$ $\left(10^{-4} \mathrm{M}\right)+$ proline $(10 \mathrm{mM})$ and $\mathrm{T}_{6}=\mathrm{L}-\mathrm{TRP}\left(10^{-4} \mathrm{M}\right)+$ proline $\left.(20 \mathrm{mM})\right]$. The experimental results revealed that growth and photosynthetic efficiency of wheat were decreased due to reduced water supply. However, exogenously applied proline and L-TRP considerably ameliorated the effect of drought stress. Combined application of L-TRP $\left(10^{-4} \mathrm{M}\right)$ and proline $(20 \mathrm{mM})$ showed better results and induced tolerance to drought stress, in comparison with other treatments. Foliar application of proline and L-TRP also enhanced the photosynthetic rate which might be related with the improved photosynthetic pigments. Overall, exogenously applied proline and L-TRP mitigated the adverse effects of moisture deficit on growth and photosynthetic efficiency of wheat crop.
\end{abstract}

Keywords: wheat, drought, proline, L-tryptophan, foliar application

\section{Introduction}

Wheat (Triticum aestivum L.) is an important cereal crop Worldwide. It is consumed as a staple food by one third of global population (Charkazi et al., 2010). Wheat is one of the most important energy sources for exorbitantly increasing population of the World (Debasis and Paramjit, 2003). Nonetheless, shortage of water, owing to climate variations, is an emerging issue in food production (Flexas et al., 2013). Among several abiotic factors which reduce the yield of wheat, drought is of prime importance. Scarcity of water is the major cause of variation in wheat yields in several regions of the World (Raza et al., 2012). A considerable part of

*Author for correspondence;

E-mail: bukhariasad@yahoo.com agricul-tural land is severely affected by drought strain in the World which means drought stress is one of the most destructive ecological phenomena, depressing wheat yield Worldwide (Jamil et al., 2018). In Pakistan wheat is grown under extremely varying conditions of soil and climate. One third of the total area under wheat cultivation is rain-fed with uncommon rainfall (Khanzada et al., 2001). Limited water supply reduces leaf area, root development and stem elongation. Water utilization efficiency of the plant is also diminished due to disturbance in plant water relations (Farooq et al., 2009).

Osmolytes have been proved to serve as protective medium under stress and can be used very competently in crop plants for alleviating the effects of different 
kinds of abiotic stresses like drought (Bowne et al., 2012). They also concluded that proline activates several enzymes with a varied range of thermal sensitivities, and from different sources, against the in vivo inactivating effects of heat. A number of solutes (D-proline, hydroxyl proline, glycine, valine and glycerol), in addition to proline, demonstrate this stress ameliorating effect on plants. Hence it is concluded that the interaction between solute and enzyme protein is a general one with respect to both effecter and receptor molecules. The role of proline under drought stress includes osmotic adjustments, protection of membranes and enzyme system, scavenging of reactive oxygen species and conservation of energy and nitrogen to be utilized during exposure to abiotic stresses.

L-Tryptophan (L-TRP) is an essential unit for animals, plants and some bacteria (Meister, 1965). It is a precursors for a wide range of secondary metabolites (Less and Galili, 2008). It is considered as the main precursor for the synthesis of auxins in plant body, which plays a pivotal role in regulating the plant's growth and developmental processes (Wang et al., 2008). Several microorganisms release secondary metabolites upon the use of L-TRP. Being physiological precursor of auxins, L-tryptophan has more affirmative impact on growth and yield of plants than pure auxins (Zahir et al., 1999). A number of studies have reported the ameliorative role of L-TRP in improving physiological process of plants under different types of environmental stresses (Rao et al., 2012).

\section{Materials and Methods}

Site, experimental design and treatments. The current investigations were carried out during 2015-2016 at Agricultural Research Farm of the University College of Agriculture and Environmental Sciences, The Islamia University of Bahawalpur, Pakistan. It is situated on $29^{\circ} \mathrm{N}$ and $73^{\circ} \mathrm{E}$. Bahawalpur is situated at the altitude of $159 \mathrm{~m}$ and has been characterized with very hot and cold climate during summer and winter, respectively. However, drought is a permanent feature of this region, throughout the year, except occasional showers. The maximum temperature rises to $48^{\circ} \mathrm{C}$ while, minimum temperature falls to $7{ }^{\circ} \mathrm{C}$. The trial was replicated thrice using randomized complete block design having split plot system. Drought stress treatments were allotted to main plots and foliar application of proline and Ltryptophan was allotted to sub plots. The net sub-plot size was $5 \mathrm{~m} \times 3 \mathrm{~m}$. Drought stress was given in three levels $\left(\mathrm{I}_{1}=\right.$ control; $\mathrm{I}_{2}=$ drought stress at tillering stage and $\mathrm{I}_{3}=$ drought stress at grain filling stage) while, foliar application of proline and L-TRP was done in six levels $\left[\mathrm{T}_{1}=\right.$ control; $\mathrm{T}_{2}=$ proline $(10 \mathrm{mM}) ; \mathrm{T}_{3}=$ proline $(20 \mathrm{mM}) ; \mathrm{T}_{4}=\mathrm{L}-\mathrm{TRP}\left(10^{-4} \mathrm{M}\right) ; \mathrm{T}_{5}=\mathrm{L}-\mathrm{TRP}\left(10^{-4} \mathrm{M}\right)$ + proline $(10 \mathrm{mM})$ and $\mathrm{T}_{6}=\mathrm{L}-\mathrm{TRP}\left(10^{-4} \mathrm{M}\right)+$ proline (20 mM)].

Data recording and analysis. Observations pertaining to growth and yield related traits were recorded according to standard procedures. Data on plant height was recorded by measuring randomly selected 5 plants in each subplot from base of plant to the tip of spikes including awns at physiological maturity. Fertile tillers $\left(\mathrm{m}^{-2}\right)$ were counted from an area of $1 \mathrm{~m}^{2}$ at 3 randomly selected locations in each subplot at the time of harvest. Spike length $(\mathrm{cm})$ was recorded by measuring randomly selected 5 spikes in each subplot. Number of spikelets per spike was recorded by counting the spikelets on randomly selected 5 spikes in each subplot. 1000 grains, collected from each subplot, were weighed using electronic balance to determine 1000 grain weight. Biological yield was calculated by weighing sundried plants per plot before threshing, followed by conversion in tons per hectare. Straw yield per plot was recorded and converted into tons per hectare. After threshing grain yield per plot was measured and converted into tons per hectare. Harvest index (\%) was determined as described below:

$$
\text { Harvest index }(\%)=\frac{(\text { Economic yield }(\text { grains })}{(\text { Biological yield })} \times 100
$$

Chlorophylls ' $a$ ' and ' $b$ ' contents were analyzed as described by Lichtenthaler (1987). Fresh leaves (0.2 g) were excised and placed over-night with acetone $(80 \%)$ at $4{ }^{\circ} \mathrm{C}$, followed by centrifugation of the extract at $10,000 \times \mathrm{g}$ for $5 \mathrm{~min}$. Spectrophotometer (HitachiU2001, Tokyo, Japan) was used to record the absorbance of the supernatant at 645,663 and $480 \mathrm{~nm}$. The chlorophylls ' $a$ ' and ' $b$ ' contents were determined as described below:

$$
\begin{aligned}
& \text { Chlorophyll a }(\mu \mathrm{g} / \mathrm{g})=[(12.7 \times \mathrm{OD} \text { at } 663)- \\
& (2.69 \times \mathrm{OD} \text { at } 645)] \\
& \text { Chlorophyll } \mathrm{b}(\mu \mathrm{g} / \mathrm{g})=[(22.9 \times \mathrm{OD} \text { at } 645)- \\
& (4.68 \times \text { OD at } 663)]
\end{aligned}
$$

Flame photometer (Jenway, PFP-7) was employed to quantify the concentration of $\mathrm{Na}^{+}$and $\mathrm{K}^{+}$cations. Standard curves were obtained by using the standard 
solutions of $\mathrm{Na}^{+}$and $\mathrm{K}^{+}$( 5 to $25 \mathrm{mg} / \mathrm{L}$ ). The samples were run on flame photometer and the concentrations of $\mathrm{Na}^{+}$and $\mathrm{K}^{+}$were measured by comparing with the standard curves.

Leaf relative water content (RWC) was measured using the method described by Santos and Silva, (2015). Fresh weight of completely extended leaves was recorded immediately after their removal from the plants followed by soaking in distilled water for $4 \mathrm{~h}$ under a constant light at room temperature. The turgid weight of leaf was calculated and the samples were placed in oven for drying at $80^{\circ} \mathrm{C}$ for $24 \mathrm{~h}$. The dried samples were weighed and the relative water contents of the leaf were calculated by employing follow equation:

$$
\mathrm{RWC}=\frac{(\text { Fresh weight }- \text { Dry weight })}{(\text { Turged weight-Dry weight })} \times 100
$$

Data collected was statistically analyzed by a computer program STATISTIX. Least significant difference test (LSD) at 0.05 probability level was employed to compare the difference among the treatment means (Steel et al., 1997).

\section{Results and Discussion}

Plant height, productive tillers and spike length. Data regarding plant height, productive tillers and spike length are shown in Table 1. Both treatments (reduced irrigation and spray of osmolytes) markedly affected plant height, number of productive tillers $/ \mathrm{m}^{2}$ and spike length. Highest value of plant height was observed in control irrigation $\left(\mathrm{I}_{1}\right)$ when sprayed with $20 \mathrm{mM}$ proline $\left(\mathrm{T}_{3}\right), 10^{-4} \mathrm{M} \mathrm{L}$-TRP $\left(\mathrm{T}_{4}\right), \mathrm{L}-\mathrm{TRP}+10 \mathrm{mM}$ proline $\left(\mathrm{T}_{5}\right)$ and $\mathrm{L}-\mathrm{TRP}+20 \mathrm{mM}$ proline $\left(\mathrm{T}_{6}\right)$. At tillering and grain filling stages, the limited supply of irrigation considerably reduced the plant height. The plants exposed to drought stress at tillering stage exhibited the lowest value of plant height without the foliar application of osmolytes. The spray of osmolytes ameliorated the deleterious effect of drought stress at both stages. However, the application of L-TRP and Proline together proved to be more effective as compared with their individual treatment (Table 1). Data on productive tillers shows that the highest value was obtained in control irrigation treatment with foliar application of osmolytes. The maximum value of productive tillers was noted in drought stress at tillering stage when sprayed with

Table 1. Effect of drought stress and osmolytes on plant height, productive tillers and spike length of wheat (Triticum aestivum L.)

\begin{tabular}{lllll}
\hline \hline Drought level & Osmolyte treatment & Plant height & Productive tillers $/ \mathrm{m}^{2}$ & Spike length(cm) \\
\hline $\mathrm{I}_{1}$ & $\mathrm{~T}_{1}$ & $98.60 \mathrm{ab}$ & $296.67 \mathrm{a}-\mathrm{c}$ & $11.67 \mathrm{a}-\mathrm{c}$ \\
& $\mathrm{T}_{2}$ & $99.03 \mathrm{ab}$ & $302.27 \mathrm{ab}$ & $11.87 \mathrm{a}-\mathrm{c}$ \\
& $\mathrm{T}_{3}$ & $99.80 \mathrm{a}$ & $306.83 \mathrm{ab}$ & $12.17 \mathrm{ab}$ \\
& $\mathrm{T}_{4}$ & $99.77 \mathrm{a}$ & $309.67 \mathrm{ab}$ & $12.30 \mathrm{ab}$ \\
& $\mathrm{T}_{5}$ & $101.83 \mathrm{a}$ & $310.47 \mathrm{a}$ & $12.57 \mathrm{ab}$ \\
& $\mathrm{T}_{6}$ & $103.00 \mathrm{a}$ & $319.13 \mathrm{a}$ & $12.73 \mathrm{a}$ \\
$\mathrm{I}_{2}$ & $\mathrm{~T}_{1}$ & $80.33 \mathrm{f}$ & $280.50 \mathrm{a}-\mathrm{c}$ & $10.10 \mathrm{c}$ \\
& $\mathrm{T}_{2}$ & $81.63 \mathrm{f}$ & $282.90 \mathrm{a}-\mathrm{c}$ & $10.33 \mathrm{bc}$ \\
& $\mathrm{T}_{3}$ & $87.00 \mathrm{e}$ & $285.23 \mathrm{a}-\mathrm{c}$ & $10.43 \mathrm{bc}$ \\
& $\mathrm{T}_{4}$ & $89.63 \mathrm{c}-\mathrm{e}$ & $272.57 \mathrm{c}$ & $10.80 \mathrm{bc}$ \\
& $\mathrm{T}_{5}$ & $91.37 \mathrm{c}-\mathrm{e}$ & $275.83 \mathrm{bc}$ & $10.97 \mathrm{bc}$ \\
& $\mathrm{T}_{6}$ & $93.23 \mathrm{c}$ & $275.83 \mathrm{bc}$ & $11.50 \mathrm{a}-\mathrm{c}$ \\
$\mathrm{I}_{3}$ & $\mathrm{~T}_{1}$ & $88.23 \mathrm{de}$ & $276.43 \mathrm{bc}$ & $10.30 \mathrm{bc}$ \\
& $\mathrm{T}_{2}$ & $87.13 \mathrm{e}$ & $281.03 \mathrm{a}-\mathrm{c}$ & $10.50 \mathrm{bc}$ \\
& $\mathrm{T}_{3}$ & $89.67 \mathrm{c}-\mathrm{e}$ & $281.73 \mathrm{a}-\mathrm{c}$ & $10.73 \mathrm{bc}$ \\
& $\mathrm{T}_{4}$ & $89.97 \mathrm{c}-\mathrm{e}$ & $283.43 \mathrm{a}-\mathrm{c}$ & $11.03 \mathrm{a}-\mathrm{c}$ \\
& $\mathrm{T}_{5}$ & $92.73 \mathrm{~cd}$ & $289.97 \mathrm{a}-\mathrm{c}$ & $11.13 \mathrm{a}-\mathrm{c}$ \\
& $\mathrm{T}_{6}$ & $94.23 \mathrm{bc}$ & $293.70 \mathrm{a}-\mathrm{c}$ & $11.43 \mathrm{a}-\mathrm{c}$ \\
\hline \hline
\end{tabular}

Means sharing the common letters are statistically non-significant at $5 \%$ probability level $(\mathrm{n}=3) . \mathrm{I}_{1}=$ control (normal irrigation); $\mathrm{I}_{2}=$ drought stress at tillering stage; $\mathrm{I}_{3}=$ drought stress at grain filling stage; $\mathrm{T}_{1}=$ control (distill water spray); $\mathrm{T}_{2}=$ proline $(10 \mathrm{mM}) ; \mathrm{T}_{3}=$ proline $(20 \mathrm{mM}) ; \mathrm{T}_{4}=\mathrm{L}-\mathrm{TRP}\left(10^{-4} \mathrm{M}\right) ; \mathrm{T}_{5}=\mathrm{L}-\mathrm{TRP}\left(10^{-4} \mathrm{M}\right)+$ proline $(10 \mathrm{mM})$ and $\mathrm{T}_{6}=\mathrm{L}-\mathrm{TRP}\left(10^{-4} \mathrm{M}\right)+$ proline $(20 \mathrm{mM})$. 
proline $20 \mu \mathrm{m}$. However, combined treatment of proline and L-TRP mitigated the impact of drought and enhanced the tillering capacity of the plants, as compared with the control. The data concerning spike length revealed that drought stress caused a significant decline in the value. However, individual as well as combined application of osmolytes caused a significant enhancement in spike length (Table 1).

Photosynthetic pigments. Data regarding chlorophyll $a$ and $b$ contents are shown in Table 2 . Both treatments; foliar application of osmolytes and irrigation levels significantly affected chlorophyll a and b contents. Drought stress at both stages (tillering and grain filling) significantly reduced chlorophyll $\mathrm{a}$ and $\mathrm{b}$ contents. However, foliar application of proline and L-TRP mitigated the impact of reduced moisture supply and increased the contents of photosynthetic pigments (Table 2).

Table 2. Effect of drought stress and osmolytes on photosynthetic pigments of wheat (Triticum aestivum L.)

\begin{tabular}{llll}
\hline $\begin{array}{l}\text { Drought } \\
\text { level }\end{array}$ & $\begin{array}{l}\text { Osmolyte } \\
\text { treatment }\end{array}$ & $\begin{array}{l}\text { chlorophyll } \\
\text { (a) contents } \\
(\mu \mathrm{g} / \mathrm{g} \text { fresh } \\
\text { weight) }\end{array}$ & $\begin{array}{l}\text { chlorophyll } \\
(\mathrm{b}) \text { contents } \\
(\mu \mathrm{g} / \mathrm{g} \text { fresh } \\
\text { weight) }\end{array}$ \\
\hline $\mathrm{I}_{1}$ & $\mathrm{~T}_{1}$ & $0.963 \mathrm{~g}$ & $0.04 \mathrm{a}$ \\
& $\mathrm{T}_{2}$ & $1.07 \mathrm{fg}$ & $0.03 \mathrm{ab}$ \\
& $\mathrm{T}_{3}$ & $1.50 \mathrm{a}$ & $0.01 \mathrm{ab}$ \\
& $\mathrm{T}_{4}$ & $1.07 \mathrm{fg}$ & $0.04 \mathrm{a}$ \\
& $\mathrm{T}_{5}$ & $1.10 \mathrm{e}-\mathrm{g}$ & $0.03 \mathrm{ab}$ \\
& $\mathrm{T}_{6}$ & $1.32 \mathrm{~b}-\mathrm{d}$ & $0.03 \mathrm{ab}$ \\
$\mathrm{I}_{2}$ & $\mathrm{~T}_{1}$ & $1.28 \mathrm{c}-\mathrm{e}$ & $0.02 \mathrm{ab}$ \\
& $\mathrm{T}_{2}$ & $1.47 \mathrm{ab}$ & $0.01 \mathrm{ab}$ \\
& $\mathrm{T}_{3}$ & $1.45 \mathrm{a}-\mathrm{c}$ & $0.003 \mathrm{~b}$ \\
& $\mathrm{~T}_{4}$ & $1.56 \mathrm{a}$ & $0.008 \mathrm{ab}$ \\
& $\mathrm{T}_{5}$ & $1.44 \mathrm{a}-\mathrm{c}$ & $0.01 \mathrm{ab}$ \\
& $\mathrm{T}_{6}$ & $1.05 \mathrm{fg}$ & $0.02 \mathrm{ab}$ \\
$\mathrm{I}_{3}$ & $\mathrm{~T}_{1}$ & $0.969 \mathrm{~g}$ & $0.04 \mathrm{a}$ \\
& $\mathrm{T}_{2}$ & $1.22 \mathrm{~d}-\mathrm{f}$ & $0.02 \mathrm{ab}$ \\
& $\mathrm{T}_{3}$ & $1.40 \mathrm{a}-\mathrm{c}$ & $0.02 \mathrm{ab}$ \\
& $\mathrm{T}_{4}$ & $1.01 \mathrm{~g}$ & $0.03 \mathrm{ab}$ \\
& $\mathrm{T}_{5}$ & $1.12 \mathrm{e}-\mathrm{g}$ & $0.02 \mathrm{ab}$ \\
& $\mathrm{T}_{6}$ & $1.00 \mathrm{~g}$ & $0.03 \mathrm{ab}$ \\
\hline \hline & & & \\
& & &
\end{tabular}

Means sharing the common letters are statistically nonsignificant at $5 \%$ probability level $(n=3) . I_{1}=$ control (normal irrigation); $\mathrm{I}_{2}=$ drought stress at tillering stage; $\mathrm{I}_{3}=$ drought stress at grain filling stage; $\mathrm{T}_{1}=$ control (distill water spray); $\mathrm{T}_{2}=$ proline $(10 \mathrm{mM}) ; \mathrm{T}_{3}=$ proline $(20 \mathrm{mM}) ; \mathrm{T}_{4}=\mathrm{L}-\mathrm{TRP}$ $\left(10^{-4} \mathrm{M}\right) ; \mathrm{T}_{5}=\mathrm{L}$-TRP $\left(10^{-4} \mathrm{M}\right)+$ proline $(10 \mathrm{mM})$ and $\mathrm{T}_{6}=$ $\mathrm{L}$-TRP $\left(10^{-4} \mathrm{M}\right)+$ proline $(20 \mathrm{mM})$.
Sodium, potassium and relative water contents. Data regarding $\mathrm{Na}$ and $\mathrm{K}$ reveals that drought stress increased $\mathrm{Na}^{+}$and decreased $\mathrm{K}^{+}$contents. The values of $\mathrm{Na}^{+}$and $\mathrm{K}^{+}$were significantly decreased and increased, respectively, by foliar application of osmolytes individually as well as in combination (Table 3). Both treatments; foliar application of osmolytes and limited moisture supply, revealed a significant effect on $\mathrm{Na}^{+}$and $\mathrm{K}^{+}$ contents in leaves. $\mathrm{T}_{4} \mathrm{I}_{3}$ gave the highest value (49.48 ppm) of $\mathrm{Na}^{+}$contents in leaves and $\mathrm{T}_{5} \mathrm{I}_{3}$ gave the highest value $(17.67 \mathrm{ppm})$ of $\mathrm{K}^{+}$contents in leaves. Minimum value (45.67 ppm) of $\mathrm{Na}^{+}$contents in leaves was observed in $\mathrm{T}_{1} \mathrm{I}_{1}$ which was $7.43 \%$ less than the highest value and the minimum value $(15.00 \mathrm{ppm})$ of $\mathrm{K}^{+}$content in leaves was observed in $\mathrm{T}_{3} \mathrm{I}_{1}$ which was $15.09 \%$ less than the highest one.

Data regarding relative water contents in leaves (\%) is shown in the Table 3. Both treatments; foliar application

Table 3. Effect of drought stress and osmolytes on sodium, potassium and relative water contents of wheat (Triticum aestivum L.)

\begin{tabular}{|c|c|c|c|c|}
\hline $\begin{array}{l}\text { Drought } \\
\text { level }\end{array}$ & $\begin{array}{l}\text { Osmolyte } \\
\text { treatment } \\
(\mathrm{ppm})\end{array}$ & $\begin{array}{l}\text { Sodium } \\
\text { content } \\
(\mathrm{ppm})\end{array}$ & $\begin{array}{l}\text { Potassium } \\
\text { content } \\
(\%)\end{array}$ & $\begin{array}{l}\text { Relative water } \\
\text { content in } \\
\text { leaves }\end{array}$ \\
\hline \multirow[t]{6}{*}{$\mathrm{I}_{1}$} & $\mathrm{~T}_{1}$ & $45.67 b$ & $16.33 \mathrm{ab}$ & $63.13 \mathrm{a}-\mathrm{d}$ \\
\hline & $\mathrm{T}_{2}$ & $46.43 \mathrm{ab}$ & $16.67 \mathrm{ab}$ & $74.63 a-c$ \\
\hline & $\mathrm{T}_{3}$ & $46.67 \mathrm{ab}$ & $15.00 \mathrm{~b}$ & $70.83 a-c$ \\
\hline & $\mathrm{T}_{4}$ & $46.67 \mathrm{ab}$ & $16.33 \mathrm{ab}$ & $75.97 \mathrm{a}-\mathrm{c}$ \\
\hline & $\mathrm{T}_{5}$ & $49.28 \mathrm{a}$ & $15.67 \mathrm{ab}$ & $79.17 \mathrm{a}-\mathrm{c}$ \\
\hline & $\mathrm{T}_{6}$ & $48.67 \mathrm{ab}$ & $15.33 \mathrm{ab}$ & $84.13 \mathrm{ab}$ \\
\hline \multirow[t]{6}{*}{$\mathrm{I}_{2}$} & $\mathrm{~T}_{1}$ & $49.17 \mathrm{a}$ & $15.33 \mathrm{ab}$ & $44.60 \mathrm{c}$ \\
\hline & $\mathrm{T}_{2}$ & $48.51 \mathrm{ab}$ & $16.33 \mathrm{ab}$ & $50.93 \mathrm{~cd}$ \\
\hline & $\mathrm{T}_{3}$ & 48.29ab & $16.00 \mathrm{ab}$ & $55.93 \mathrm{~b}-\mathrm{d}$ \\
\hline & $\mathrm{T}_{4}$ & $49.39 \mathrm{a}$ & $16.00 \mathrm{ab}$ & $55.80 \mathrm{~b}-\mathrm{d}$ \\
\hline & $\mathrm{T}_{5}$ & $49.34 \mathrm{a}$ & $15.33 \mathrm{ab}$ & $62.80 \mathrm{a}-\mathrm{d}$ \\
\hline & $\mathrm{T}_{6}$ & $47.39 \mathrm{ab}$ & $17.00 \mathrm{ab}$ & $66.83 a-d$ \\
\hline \multirow[t]{6}{*}{$\mathrm{I}_{3}$} & $\mathrm{~T}_{1}$ & $49.32 \mathrm{a}$ & $15.00 \mathrm{~b}$ & $40.13 d$ \\
\hline & $\mathrm{T}_{2}$ & $48.41 \mathrm{ab}$ & $16.67 \mathrm{ab}$ & $57.20 \mathrm{~b}-\mathrm{d}$ \\
\hline & $\mathrm{T}_{3}$ & $46.53 \mathrm{ab}$ & $16.00 \mathrm{ab}$ & $61.00 \mathrm{a}-\mathrm{d}$ \\
\hline & $\mathrm{T}_{4}$ & $49.48 \mathrm{a}$ & $16.33 \mathrm{ab}$ & $61.67 \mathrm{a}-\mathrm{d}$ \\
\hline & $\mathrm{T}_{5}$ & $48.33 \mathrm{ab}$ & $17.67 \mathrm{a}$ & $63.10 \mathrm{a}-\mathrm{d}$ \\
\hline & $\mathrm{T}_{6}$ & $48.09 \mathrm{ab}$ & $17.67 \mathrm{a}$ & $69.87 \mathrm{a}-\mathrm{d}$ \\
\hline
\end{tabular}

Means sharing the common letters are statistically nonsignificant at $5 \%$ probability level $(n=3)$. $I_{1}=$ control (normal irrigation); $\mathrm{I}_{2}=$ drought stress at tillering stage; $\mathrm{I}_{3}=$ drought stress at grain filling stage; $\mathrm{T}_{1}=$ control (distill water spray); $\mathrm{T}_{2}=$ proline $(10 \mathrm{mM}) ; \mathrm{T}_{3}=$ proline $(20 \mathrm{mM}) ; \mathrm{T}_{4}=\mathrm{L}-\mathrm{TRP}$ $\left(10^{-4} \mathrm{M}\right) ; \mathrm{T}_{5}=\mathrm{L}-\mathrm{TRP}\left(10^{-4} \mathrm{M}\right)+$ proline $(10 \mathrm{mM})$ and $\mathrm{T}_{6}=$ L-TRP $\left(10^{-4} \mathrm{M}\right)+$ proline $(20 \mathrm{mM})$. 
of osmolytes and irrigation levels showed significant effect on RWC in leaves. Among the interactions, $T_{6} I_{1}$ produced the highest value $(84.13 \%)$ of leaf RWC. Nonetheless, the minimum value (40.13) leaf RWC was observed in $\mathrm{T}_{1} \mathrm{I}_{3}$ which was $52.35 \%$ less than the highest one. Individual as well as combined application of osmolytes considerably improved the relative water content of leaves under reduced irrigation.

Biological yield of seed and straw, harvest index and 1000 grain weight. Data on yield attributes are presented in Table 4. Biological yield was severely declined by drought stress at both growth stages under study (tillering and grain filling). Minimum value of biological yield was recorded in those plots where drought stress was imposed at grain filling stage followed by drought stress at tillering stage, without the application of osmolytes. However, foliar application of osmolytes individually, as well as in combination ameliorated the stress and significantly enhanced the biological yield. Maximum biological yield was noted under normal irrigation when sprayed with both osmolytes (L-TRP $+20 \mathrm{mM}$ proline) in $\mathrm{T}_{6} \mathrm{I}_{1}$.
Grain yield also exhibited the similar behaviour. Minimum grain yield was recorded under drought stress at grain filling stage followed by tillering, without foliar application of osmolytes. Individual as well as combined treatment of plants with proline and L-TRP, mitigated the drought stress and caused a considerable enhancement in grain yield. Maximum grain yield was recorded in normal irrigation under combined application of both osmolytes (L-TRP $+10 \mathrm{mM}$ proline). Straw yield showed the similar behavior as that of biological yield and grain yield.

Harvest index was severely reduced by drought stress at tillering as well as grain filling stage. The highest reduction in harvest index was noted in those plants where drought stress was imposed at grain filling stage without the spray of osmolytes, while lowest reduction was observed under normal irrigation with combined application of L-TRP and $20 \mathrm{mM}$ proline $\mathrm{T}_{6} \mathrm{I}_{1}$. Nonetheless, application of osmolytes under drought stress at both stages (Tillering and grain filling) improved the harvest index.

Table 4. Effect of drought stress and osmolytes on biological yield, grain yield, straw yield, harvest index and 1000 grain weight of wheat (Triticum aestivum L.)

\begin{tabular}{cllllll}
\hline \hline Drought level & Osmolyte treatment & $\begin{array}{l}\text { Biological yield } \\
(\mathrm{t} / \mathrm{ha})\end{array}$ & $\begin{array}{l}\text { Grain yield } \\
(\mathrm{t} / \mathrm{ha})\end{array}$ & $\begin{array}{l}\text { Straw yield } \\
(\mathrm{t} / \mathrm{ha})\end{array}$ & $\begin{array}{l}\text { Harvest index } \\
(\%)\end{array}$ & $\begin{array}{l}1000 \text { grain weight } \\
(\mathrm{g})\end{array}$ \\
\hline $\mathrm{I}_{1}$ & $\mathrm{~T}_{1}$ & $10.50 \mathrm{a}-\mathrm{c}$ & $4.33 \mathrm{ac}$ & $6.47 \mathrm{a}-\mathrm{c}$ & $44.1 \mathrm{a}-\mathrm{c}$ & $48.33 \mathrm{~b}-\mathrm{d}$ \\
& $\mathrm{T}_{2}$ & $10.30 \mathrm{a}-\mathrm{c}$ & $4.90 \mathrm{ac}$ & $6.37 \mathrm{a}-\mathrm{c}$ & $47.89 \mathrm{a}-\mathrm{c}$ & $46.00 \mathrm{c}-\mathrm{e}$ \\
& $\mathrm{T}_{3}$ & $10.43 \mathrm{a}-\mathrm{c}$ & $5.03 \mathrm{ac}$ & $6.13 \mathrm{a}-\mathrm{c}$ & $48.26 \mathrm{ab}$ & $51.00 \mathrm{a}-\mathrm{c}$ \\
& $\mathrm{T}_{4}$ & $11.07 \mathrm{ab}$ & $5.16 \mathrm{ab}$ & $6.17 \mathrm{a}-\mathrm{c}$ & $46.69 \mathrm{a}-\mathrm{c}$ & $51.33 \mathrm{a}-\mathrm{c}$ \\
& $\mathrm{T}_{5}$ & $11.77 \mathrm{ab}$ & $5.36 \mathrm{a}$ & $6.83 \mathrm{ab}$ & $45.65 \mathrm{a}-\mathrm{c}$ & $53.33 \mathrm{ab}$ \\
$\mathrm{I}_{2}$ & $\mathrm{~T}_{6}$ & $13.13 \mathrm{a}$ & $4.6 \mathrm{a}-\mathrm{c}$ & $7.97 \mathrm{a}$ & $51.29 \mathrm{a}$ & $56.33 \mathrm{a}$ \\
& $\mathrm{T}_{1}$ & $8.07 \mathrm{~b}-\mathrm{d}$ & $3.13 \mathrm{bc}$ & $4.03 \mathrm{~d}$ & $42.44 \mathrm{a}-\mathrm{c}$ & $41.00 \mathrm{ef}$ \\
& $\mathrm{T}_{2}$ & $8.53 \mathrm{a}-\mathrm{d}$ & $3.23 \mathrm{bc}$ & $4.70 \mathrm{~b}-\mathrm{d}$ & $38.40 \mathrm{a}-\mathrm{c}$ & $41.33 \mathrm{ef}$ \\
& $\mathrm{T}_{3}$ & $8.03 \mathrm{~cd}$ & $3.37 \mathrm{bc}$ & $4.67 \mathrm{a}-\mathrm{d}$ & $42.26 \mathrm{a}-\mathrm{c}$ & $43.00 \mathrm{def}$ \\
& $\mathrm{T}_{4}$ & $8.93 \mathrm{a}-\mathrm{d}$ & $3.37 \mathrm{bc}$ & $5.13 \mathrm{a}-\mathrm{d}$ & $35.51 \mathrm{bc}$ & $46.00 \mathrm{c}-\mathrm{e}$ \\
& $\mathrm{T}_{5}$ & $9.50 \mathrm{a}-\mathrm{d}$ & $3.92 \mathrm{bc}$ & $5.28 \mathrm{a}-\mathrm{d}$ & $41.56 \mathrm{a}-\mathrm{c}$ & $47.00 \mathrm{c}-\mathrm{e}$ \\
& $\mathrm{T}_{6}$ & $9.87 \mathrm{a}-\mathrm{d}$ & $4.02 \mathrm{a}-\mathrm{c}$ & $5.85 \mathrm{a}-\mathrm{d}$ & $41.07 \mathrm{a}-\mathrm{c}$ & $48.33 \mathrm{~b}-\mathrm{d}$ \\
& $\mathrm{T}_{3}$ & $6.57 \mathrm{~d}$ & $2.80 \mathrm{c}$ & $4.10 \mathrm{~cd}$ & $33.47 \mathrm{c}$ & $38.67 \mathrm{f}$ \\
& $\mathrm{T}_{2}$ & $8.27 \mathrm{a}-\mathrm{d}$ & $3.27 \mathrm{bc}$ & $5.00 \mathrm{ad}$ & $39.22 \mathrm{a}-\mathrm{c}$ & $44.67 \mathrm{~d}-\mathrm{f}$ \\
& $\mathrm{T}_{3}$ & $8.00 \mathrm{~cd}$ & $3.30 \mathrm{bc}$ & $4.50 \mathrm{~b}-\mathrm{d}$ & $42.49 \mathrm{a}-\mathrm{c}$ & $42.67 \mathrm{~d}-\mathrm{f}$ \\
& $\mathrm{T}_{4}$ & $8.57 \mathrm{a}-\mathrm{d}$ & $3.27 \mathrm{bc}$ & $5.13 \mathrm{a}-\mathrm{d}$ & $34.95 \mathrm{bc}$ & $43.33 \mathrm{~d}-\mathrm{f}$ \\
& $\mathrm{T}_{5}$ & $9.50 \mathrm{a}-\mathrm{d}$ & $3.90 \mathrm{bc}$ & $5.60 \mathrm{a}-\mathrm{d}$ & $41.13 \mathrm{a}-\mathrm{c}$ & $47.33 \mathrm{c}-\mathrm{e}$ \\
& $\mathrm{T}_{6}$ & $10.00 \mathrm{a}-\mathrm{c}$ & $4.10 \mathrm{a}-\mathrm{c}$ & $5.90 \mathrm{a}-\mathrm{d}$ & $40.99 \mathrm{a}-\mathrm{c}$ & $49.00 \mathrm{~b}-\mathrm{d}$ \\
\hline \hline
\end{tabular}

Means sharing the common letters are statistically non-significant at $5 \%$ probability level $(n=3)$. $I_{1}=$ control (normal irrigation); $\mathrm{I}_{2}=$ drought stress at tillering stage; $\mathrm{I}_{3}=$ drought stress at grain filling stage; $\mathrm{T}_{1}=$ control (distill water spray); $\mathrm{T}_{2}=$ proline $(10 \mathrm{mM}) ; \mathrm{T}_{3}=$ proline $(20 \mathrm{mM}) ; \mathrm{T}_{4}=\mathrm{L}-\mathrm{TRP}\left(10^{-4} \mathrm{M}\right) ; \mathrm{T}_{5}=\mathrm{L}-\mathrm{TRP}\left(10^{-4} \mathrm{M}\right)+$ proline $(10 \mathrm{mM})$ and $\mathrm{T}_{6}=\mathrm{L}-\mathrm{TRP}\left(10^{-4} \mathrm{M}\right)+$ proline $(20 \mathrm{mM})$. 
Drought stress at both developmental stages caused a pronounced decline in 1000-grain weight. Maximum reduction in 100-grain weight was induced by drought stress at grain filling stage. However, application of osmolytes significantly improved the value under drought stress at both growth stages (tillering and grain filling).

Plant height is an important growth regulating parameter. In present study, drought stress negatively affected the plant height because water stress was given to the crop at tillering and grain filling stage, but foliar application of osmoprotectants significantly affected the plant height. Among the interactions, $\mathrm{T}_{6} \mathrm{I}_{1}[\mathrm{NPK}+$ L-tryptophan + proline $(20 \mathrm{mM})$ with controlled irrigation] gave the highest value of plant height $(103.00 \mathrm{~cm})$. Similar observations have also been reported by previous researchers (Raza et al., 2012; Ashraf and Foolad, 2007).

Number of productive tillers per $\mathrm{m}^{2}$ is directly related to the final yield. Interaction of foliar application of osmoprotectants and drought levels showed significant effect on number of productive tillers per $\mathrm{m}^{2}$. Among the interactions, $\mathrm{T}_{6} \mathrm{I}_{1}[\mathrm{NPK}+\mathrm{L}$-tryptophan + proline $(20 \mathrm{mM})$ with controlled irrigation] depicted the highest number of productive tillers per $\mathrm{m}^{2}$ (319.13). These results are also corroborated with previous findings (Raza et al., 2012; Ashraf and Foolad 2007).

Spike length is an important yield attributing trait. Yield of wheat crop is directly related to spike length as larger spikes contain more number of spikelets and ultimately the yield increases. Interaction of foliar application of osmolytes and drought levels showed significant effect on spike length $(\mathrm{cm})$. Among the interactions, $\mathrm{T}_{6} \mathrm{I}_{1}[\mathrm{NPK}$ + L-tryptophan + proline $\mathrm{P}_{2}(20 \mathrm{mM})$ with controlled irrigation] showed the highest spike length $(12.73 \mathrm{~cm})$. Similar results were found by previous scientists (Irfan et al., 2006; Abdel-Hameed et al., 2004) who reported that foliar application of osmolytes has favourable effects on growth and yield of plants.

1000-grain weight is one of the most important components of wheat crop signifying the growth and development of grains, which ultimately contributes towards the final yield. Foliar application of osmolytes significantly affected the 1000-grain weight (g). Execution of $\mathrm{T}_{6}\left[\mathrm{NPK}+\right.$ L-tryptophan + proline $\mathrm{P}_{2}(20$ $\mathrm{mM})$ ] treatment revealed the maximum 1000-grain weight $(51.22 \mathrm{~g})$. Similar findings were observed by Talaat and Youssef (2002).
Biological yield signifies the total biomass produced by the plant during its lifecycle by consumption of available inputs. Interaction of drought levels and foliar application of osmoprotectants showed significant effect on biological yield. Among the treatments, $\mathrm{T}_{6} \mathrm{I}_{1}[\mathrm{NPK}$ + L-tryptophan + proline $\mathrm{P}_{2}(20 \mathrm{mM})$ with controlled irrigation] gave the highest biological yield (13.13 t/ha). The results of the present study are in line with Ashraf and Foolad (2007).

Grain yield is the final product of the interaction of all the contributing parameters like spike length, number of spikelets per spike and finally grain weight. Any fluctuation in these components directly affects the final yield. Exogenous application of proline and L-tryptophan under drought stress increased the grain yield by reducing the harmful effects of drought. $\mathrm{T}_{5} \mathrm{I}_{1}[\mathrm{NPK}+\mathrm{L}$-tryptophan + proline $\mathrm{P}_{1}(10 \mathrm{mM})$ with controlled irrigation] produced the highest grain yield (5.36 t/ha). These results are in line with the findings of previous investigations (Irfan et al., 2006; Abdel-Hameed et al., 2004).

Potassium is an important factor for osmoregulation, stimulates enzymes of respiration and photosynthesis and plays a significant role in stomatal regulation. Concentration of potassium in leaves of wheat was decreased significantly due to reduced water supply at various growth stages. Exogenously applied proline and L-tryptophan at all growth stages significantly increased the shoot $\mathrm{K}^{+}$under stress conditions in wheat crop. However, this increase was more obvious when combination of L-tryptophan proline and was applied (a) $10^{-4} \mathrm{M}$ and $10 \mathrm{mM}$, respectively. $\mathrm{T}_{5} \mathrm{I}_{3}[\mathrm{NPK}+\mathrm{L}-$ tryptophan + proline $\mathrm{P}_{1}(10 \mathrm{mM})$ with water stress at grain filling stage] illustrated the highest value (17.67 ppm) of $\mathrm{K}^{+}$contents in leaves. Similar results have also been revealed during previous studies (Abdel-Hameed et al. 2004).

According to previous studies, chlorophyll ' $a$ ' and ' $b$ ' contents are extremely important in photochemical reactions (Santos and Silva, 2015). Water-deficit stress significantly reduced the chlorophyll ' $a$ ' and 'b' contents in present investigation. However, foliar application of osmolytes (proline and L-tryptophan) showed significant improvement in photosynthetic pigment contents. Similar findings were also observed by Ali et al. (2007) and Kaya et al. (2006).

Water-deficit stress negatively affected the relative water contents. The reduction in this attribute may be due to deficiency of chlorophyll and other photosynthetic 
pigments (Santos and Silva, 2015). Water deficit affected the leaf expansion and decreased the total surface area of leaves due to which water status of plants and relative water contents of leaf were affected. Foliar application of osmoprotectants under scarce moisture supply enhanced relative water contents in leaves. $\mathrm{T}_{6} \mathrm{I}_{1}[\mathrm{NPK}$ + proline $\mathrm{P}_{2}(20 \mathrm{mM})+$ L-tryptophan $10^{-4} \mathrm{M}$ with controlled irrigation] gave the highest value $(84.13 \%)$ of relative water contents in leaves. These results are in line with those reported by Kafi (2009).

Conflict of Interest. The authors declare no conflict of interest.

\section{References}

Abd-El-Hameed, A.M., Sarhan, S.H., Abd-El-Salam, H.Z. 2004. Evaluation of some organic acid as foliar application on growth, yield and some nutrient contents of wheat. Mansoura University Journal of Agricultural Sciences, 29: 2475-2481.

Ali, Q., Ashraf, M., Athar, H.R. 2007. Exogenously applied proline at different growth stages enhances growth of two maize cultivars grown under water deficit conditions. Pakistan Journal of Botany, 39: 1133-1144.

Ashraf, M., Foolad, M.R. 2007. Roles of glycine betaine and proline in improving plant abiotic stress resistance. Environmental and Experimental Botany, 59: 206-216.

Bowne, J.B., Erwina, T.A., Juttnerb, J., Schnurbusch, T., Langridge, P., Bacic, A., Roessner, U. 2012. Drought responses of leaf tissues from wheat cultivars of differing drought tolerance at the metabolite level. Molecular Plant, 5: 418-429.

Charkazi, F., Ramezanpour, S.S., Soltanloo, H. 2010. Expression pattern of two sugar transporter genes $\left(\mathrm{SuT}_{4}\right.$ and $\left.\mathrm{SuT}_{5}\right)$ under salt stress in wheat. Australian Journal of Crop Science. 3: 194-198.

Debasis, P., Paramjit, K.H. 2003. Genetic transformation of Indian bread (T. aestivum) and pasta (T. durum) wheat by particle bombardment of mature embryoderived calli. BioMed Central Plant Biology, 3: 35.

Farooq, M., Wahid, A., Kobayashi, N., Fujita, D., Basra, SMA. 2009. Plant drought stress: Effects, mechanisms and management. Agronomy for Sustainable Development, 29: 185-212.

Flexas, J., Niinemets, U., Galle, A., Barbour, M.M., Centritto M. 2013. Diffusional conductances to
$\mathrm{CO}_{2}$ as a target for increasing photosynthesis and photosynthetic water-use efficiency. Photosynthesis Reseasch, 117: 1-3.

Irfan, A., Basra, S.M.A., Mahammed, F., Nawaz, A. 2006. Alleviation of salinity stress in spring wheat by hormonal priming with ABA, Salicylic acid and ascorbic acid. International Journal of Agriculture and Biology, 8: 23-28.

Jamil, M., Ahmad M., Anwar, F., Zahir, Z.A., Kharal, M.A., Nazli, F. 2018. Inducing drought tolerance in wheat through combined use of L-tryptophan and Pseudomonas fluorescens. Pakistan Journal of Agricultural Sciences, 55: 331-337.

Kafi, M. 2009. The effects of salinity and light on photosynthesis, respiration and chlorophyll fluorescence in salt-tolerant and salt-sensitive wheat (Triticum aetivum L.) cultivars. Journal of Agricultural Sciences and Technology, 11: 535-547.

Kaya, M.D., Okcub, G., Ataka, M., Cikilic, Y., Kolsaricia, O. 2006. Seed treatments to overcome salt and drought stress during germination in sunflower (Helianthus annuus L.). European Journal of Agronomy, 24: 291-295.

Khanzada, B., Ashraf, M.Y., Ala, S.A., Alam, S.M., Shirazi, M.U., Ansari, R. 2001. Water relations in different Guar (Cyamopsis tetragonoloba L.) genotypes under water stress. Pakistan Journal of Botany, 33: 279-287.

Less, H., Galili, G. 2008. Principal transcriptional programs regulating plant amino acid metabolism in response to abiotic stresses. Plant Physiology, 147: 316-330.

Lichtenthaler, H.K. 1987. Chlorophylls and carotenoids: pigments of photosynthetic biomembranes. Methods in Enzymology, 148: 350-382.

Meister, A. 1965. The Role of Amino Acids in Nutrition. Biochemistry of the Amino Acids, 2nd edition, pp. 201-230, Academic Press, New York. USA.

Rao, S.R., Qayyum, A., Razzaq, A., Ahmad, M., Mahmood, I., Sher, A. 2012. Role of foliar application of salicylic acid and 1-tryptophan in drought tolerance of maize. The Journal of Animal \& Plant Sciences, 22: 768-772.

Raza, M.A.S., Saleem, M.F., Khan, I.H., Jamil, M., Ijaz, M., Khan, M.A. 2012. Evaluating the drought stress tolerance efficiency of wheat (Triticum aestivum L.) cultivars. Russian Journal of Agricultural and Socio-Economic Sciences, 12: 4146.

Santos, C.M.D., Silva, M.D.A. 2015. Physiological and 
biochemical response of sugarcane to oxidative stress induced by water deficit and paraquat. Acta Physiologiae Plantarum, 37: 172

Steel, R.G.D., Torrie, J.H., Dickey, D. 1997. Principles and Procedures of Statistics. A Biometrical Approach, pp. 352-358, $3^{\text {rd }}$ edition. Mc Graw Hill Book Co. Inc., New York. USA.

Talaat, I.M., Youssef, A.A. 2002. The role of the amino acids lysine and ornithine in growth and chemical constituents of basil plants. Egyptian Journal of Applied Sciences, 17: 83-95.

Wang, C., Yang, A., Yin, H., Zhang, J. 2008. Influence of water stress on endogenous hormone contents and cell damage of maize seedlings. Journal of Integrative Plant Biology, 50: 427-434.

Zahir, A.Z., Malik, M.A.R., Arshad, M. 1999. Effect of auxins on the growth and yield of rice. Pakistan Journal of Agricultural Sciences, 36: 3-4. 